
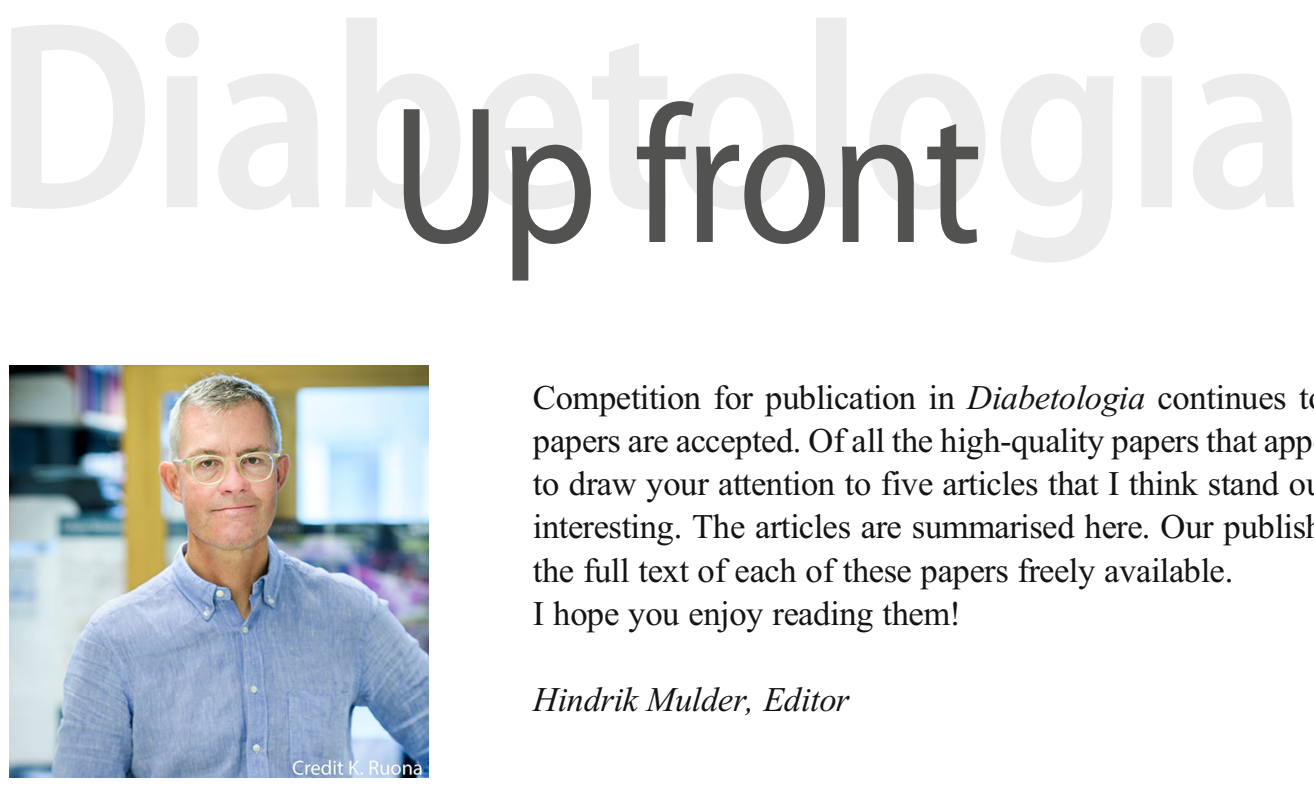

Competition for publication in Diabetologia continues to grow, and less than $20 \%$ of papers are accepted. Of all the high-quality papers that appear in this month's issue I want to draw your attention to five articles that I think stand out in some regard and are very interesting. The articles are summarised here. Our publisher, Springer, has kindly made the full text of each of these papers freely available.

I hope you enjoy reading them!

Hindrik Mulder, Editor

\section{Reducing intergenerational obesity and diabetes risk}

Katherine A. Sauder, Natalie D. Ritchie

Strategies promoting the health of women of reproductive age are urgently needed to reduce intergenerational transmission of obesity and diabetes risk. In this issue, Sauder and Ritchie (https://doi.org/10.1007/s00125-020-05341-y) summarise findings and gaps arising from published studies of prenatal and preconception lifestyle interventions. They demonstrate that prenatal interventions have shown some success in limiting gestational weight gain and reducing risk of gestational diabetes but have had little impact on offspring outcomes at birth or beyond. Preconception interventions have been successful in reducing weight and improving glucose metabolism by the end of intervention, but whether effects were sustained to conception or whether the interventions had an impact on offspring outcomes is unclear. The authors conclude that future clinical trials should include interventions with high potential for dissemination, diverse populations, thorough maternal phenotyping from enrolment through to conception and pregnancy, and rigorous assessment of offspring obesity and diabetes risks from birth onwards, including into the third generation.

The figure from this review is available as a downloadable slide
A healthy lifestyle mitigates the risk of heart disease related to type 2 diabetes: a prospective nested casecontrol study in a nationwide Swedish twin cohort

Rongrong Yang, Hui Xu, Nancy L. Pedersen, Xuerui Li, Jing Yu, Cuiping Bao, Xiuying Qi, Weili Xu

To date, epidemiological studies have shown that type 2 diabetes increases the risk of cardiovascular disease, which includes coronary heart disease and stroke. However, it is still unclear whether type 2 diabetes is specifically associated with the risk of heart disease and its subtypes, and whether a healthy lifestyle could reduce the risk of heart disease related to type 2 diabetes. In this issue, Yang, $\mathrm{Xu}$ et al (https://doi.org/10. 1007/s00125-020-05324-z) report that type 2 diabetes is associated with a more than fourfold increased risk of heart disease, including coronary heart disease, cardiac arrhythmias and heart failure. Genetic and early-life familial environmental background did not account for the association between type 2 diabetes and heart disease. However, findings suggested that adulthood lifestyle factors were important in this association: patients with type 2 diabetes who maintained a healthy lifestyle (consisting of being a non-smoker, no/ mild alcohol consumption, regular physical activity and not being overweight) had a significantly lower risk of heart disease than those with an unfavourable lifestyle. 
The authors concluded that these findings highlight the importance of a healthy lifestyle in the prevention of heart disease among individuals with type 2 diabetes.

\section{Association between diabetes and haematological malignancies: a population-based study}

Inna Y. Gong, Matthew C. Cheung, Stephanie Read, Yingbo Na, Iliana C. Lega, Lorraine L. Lipscombe

While diabetes has been implicated as a risk factor for cancer, much of the data to date have focused on solid malignancies, while less conclusive data exist for diabetes and haematological malignancies. In this issue, Gong et al (https://doi.org/10. 1007/s00125-020-05338-7) used Canadian population-based data to evaluate the incidence and prognosis of haematological malignancies among individuals with diabetes. They showed that diabetes is associated with a higher likelihood of developing a haematological malignancy. The authors also demonstrated that individuals with diabetes and haematological malignancy have higher all-cause mortality and cancerspecific mortality than those without diabetes. Importantly, these associations were seen even beyond 1 year after a diabetes diagnosis, which argues against a detection bias due to enhanced health contact. These findings suggest that diabetes adversely affects risk and prognosis of haematological malignancy, and that diabetes prevention strategies may not only reduce diabetes burden but may also potentially improve the risk of haematological malignancy.

\section{Structural and functional polarisation of human pancreatic beta cells in islets from organ donors with and without type 2 diabetes}

Louise Cottle, Wan Jun Gan, Ian Gilroy, Jaswinder S. Samra, Anthony J. Gill, Thomas Loudovaris, Helen E. Thomas, Wayne J. Hawthorne, Melkam A. Kebede, Peter Thorn

Accumulating evidence demonstrates that rodent beta cells are structurally and functional polarised. In this issue, Cottle et al (https://doi.org/10.1007/s00125-020-05345-8) investigated whether human beta cells are also polarised. This is an important question given the anatomical differences between rodent and human islets. The authors used pancreatic slices to preserve islet structure and 3D immunostaining to show that human beta cells are polarised with an apical-basal orientation, locating the basal pole of each cell at the capillary interface. Presynaptic scaffold proteins were enriched in this basal region, suggesting local specialisation for insulin granule exocytosis. The authors provide functional evidence indicating that the orientation of human beta cells is a focal adhesionmediated response to extracellular matrix proteins secreted by capillary endothelial cells. They conclude that this evidence - that human beta cells are polarised - advances our understanding of normal islet function. They state that, clinically, these findings are relevant for understanding type 2 diabetes, in which islet capillary structure changes, and for cell-based treatments for type 1 diabetes.

SUR1-mutant iPS cell-derived islets recapitulate the pathophysiology of congenital hyperinsulinism

Väinö Lithovius, Jonna Saarimäki-Vire, Diego Balboa, Hazem Ibrahim, Hossam Montaser, Tom Barsby, Timo Otonkoski

Congenital hyperinsulinism caused by mutations in the $\mathrm{K}_{\mathrm{ATP}^{-}}$ channel-encoding genes often leads to life-threatening hypoglycaemia. Current treatment options are inadequate and many patients have to undergo radical pancreatic surgery. The development of new pharmacological treatment options is hindered by the lack of disease-specific beta cell models. In this issue, Lithovius et al (https://doi.org/10.1007/s00125020-05346-7) set out to create a new disease model of congenital hyperinsulinism due to loss-of-function of the $\mathrm{K}_{\mathrm{ATP}}$ channel $\left(\mathrm{K}_{\mathrm{ATP}} \mathrm{HI}\right)$ to circumvent the issue of limited patient material. They used induced pluripotent stem cells (iPSCs), derived from an individual with $\mathrm{K}_{\mathrm{ATP}} \mathrm{HI}$, as well as CRISPR-Cas9 mutation-corrected iPSCs for control purposes. iPSCs were differentiated into pancreatic islet-like cells, allowing comparison of mutated and corrected cells, which are isogenic, both in vitro and after transplantation into mice. The authors observed insulin hypersecretion in vitro and in vivo, along with increased beta cell proliferation and mass, thus highlighting the relevance of their model for $\mathrm{K}_{\mathrm{ATP}} \mathrm{HI}$ research. They concluded that this model could serve as a platform for developing new treatment options for $\mathrm{K}_{\mathrm{ATP}} \mathrm{HI}$.

All text supplied by the authors. 